LII Zakopane School of Physics, International Symposium Breaking Frontiers, Zakopane, Poland, May 22-27, 2017

\title{
Surface Modification of Solids by Extreme Ultraviolet and Plasma Treatment
}

\author{
A. Bartnik*, H. Fiedorowicz, W. Skrzeczanowski, J. Czwartos, P. Wachulak, \\ R. JAROCKI AND J. KOSTECKI \\ Institute of Optoelectronics, Military University of Technology, S. Kaliskiego 2, 00-908 Warsaw, Poland
}

\begin{abstract}
In this paper, results of surface modification, using a laser-produced plasma source of extreme ultraviolet, and the extreme ultraviolet induced low temperature plasmas, are presented. It was shown that irradiation of different materials by intense extreme ultraviolet pulses results in strong changes of the surface morphology. Examples of micro- and nanostructures obtained this way are presented. It was also demonstrated that a dual action of the radiation pulses and low temperature plasmas allows to modify a molecular structure of exposed materials.
\end{abstract}

DOI: 10.12693/APhysPolA.133.267

PACS/topics: laser plasma, photoionized plasma, surface treatment, nanostructures

\section{Introduction}

Many materials are used in industry because of good mechanical, optical or electrical properties and their resistance to environmental factors. In case of some applications, however, surface properties of the materials have to be modified. The modification usually concerns wettability, adsorption, adhesion, optical, or other surface properties. It can be associated with changes of surface morphology or molecular structure of the near-surface layer. Various methods are used for surface structuring, including chemical [1], plasma [2], or radiation treatment. For the latter one, ultraviolet (UV) lamps or UV lasers are usually employed [3-5]. Energy of UV photons is sufficient for excitation of valence electrons of polymers. The interaction is, however, effective only for photon energies fitted to electronic resonances of molecules forming a particular polymer. In case of such fitting the absorption depth is small, affecting only the near surface layer of the order of hundreds nanometers to a few micrometers. The excited states induced in the layer can relax through radiative or non-radiative channels. One of the possible radiationless channel corresponds to bond breaking of the polymer chain. This can result in releasing of volatile fractions from the affected layer leading to smooth ablation or modification of the surface morphology. It can be also associated with changes of chemical structure and composition of the polymer molecules. For some applications irradiation is being performed in a reactive atmosphere which allows for incorporation of various functional groups into the molecular structure [4].

In this work we describe an alternative method employed for surface modification, using intense pulses of extreme ultraviolet (EUV) for material irradiation or the EUV irradiation combined with a low temperature plasma treatment. Irradiation of material surface with

*corresponding author; e-mail: abartnik@wat.edu.pl the EUV radiation results in efficient ablation, changes of surface morphology and modification of the chemical structure. Additional use of the low temperature plasma, induced by the EUV beam in a gas injected into the vicinity of the irradiated surface, allows for formation of new functional groups in the molecular structure. In the paper some examples of the surface structuring, and results of measurements of chemical changes, measured by the X-ray photoelectron spectroscopy (XPS) are presented. The photoionized plasmas employed for the surface treatment were also investigated. Typical spectra obtained in the EUV and visible (Vis) ranges for a nitrogen plasma are shown.

\section{EUV source}

In our experiments, a $10 \mathrm{~Hz}$ laser-plasma EUV source, based on a double-stream gas-puff target, irradiated with the $3 \mathrm{~ns} / 0.8 \mathrm{~J}$ Nd:YAG laser pulse, was used. Focusing conditions and plasma parameters were adjusted to obtain maximum intensity in the EUV spectral region. The radiation was focused using a gold-plated grazing incidence ellipsoidal collector, allowing for effective focusing of radiation, emitted in the wavelength range $\lambda=9 \div 70 \mathrm{~nm}$. The most intense emission was in the relatively narrow spectral region centred at $\lambda=11 \pm 1 \mathrm{~nm}$. The EUV fluence in a focal plane of the collector reached $100 \mathrm{~mJ} / \mathrm{cm}^{2}$ at the center of a focal spot in a single pulse. Detailed description of the source and parameters of focused EUV radiation can be found elsewhere [6].

\section{Photoionized plasma}

Photoionized plasmas were created by the EUV irradiation of rare or molecular gases injected into the interaction region, perpendicularly to optical axis of the irradiation system, using an auxiliary gas puff valve. The valve was equipped with a tube-shaped nozzle, $30 \mathrm{~mm}$ long with an inner diameter of $0.9 \mathrm{~mm}$. The nozzle outlet was located $2.5 \mathrm{~mm}$ from the optical axis. The gas density in the interaction region could be controlled by adjustment of either backing pressure or opening time 
of the valve. The density could be regulated within the range of the order of $1 \div 10 \%$ of the atmospheric density. The density measurements were performed using an X-ray backlighting method [7]. The EUV photons are strongly absorbed in any kind of matter including gases. $1 \mathrm{~mm}$ layer of the $\mathrm{Xe}$ or $\mathrm{SF}_{6}$ gas, having $10 \%$ of atmospheric density, absorbs almost $100 \%$ of the EUV radiation at the wavelength of $11 \mathrm{~nm}$. In case of other gases like $\mathrm{Ne}, \mathrm{O}_{2}, \mathrm{~N}_{2}$ the absorption is weaker, however, still high, of approximately 50\%. Irradiation of gases, injected into the interaction region, by EUV photons results in ionization and excitation of atoms and molecules. In case of high $Z$ atoms ( $\mathrm{Kr}, \mathrm{Xe})$ a single EUV photon carries sufficient energy for inner-shell photoionization. In case of low $Z$ atoms and molecules a sub-shell $\left(2 s^{2}\right)$ ionization is possible. In both cases a large number of electrons is released and the low temperature plasma can be formed. In case of molecular gases like $\mathrm{N}_{2}$ or $\mathrm{O}_{2}$ a dissociative ionization is one of the important processes. As a result single charged atomic ions are created. Our previous works concerned investigation of such low temperature photoionized plasmas induced in different gases. Experiments on simultaneous EUV and plasma treatment of polymers were also performed. In this work such treatment of a poly(vinylidene fluoride) (PVDF) polymer, employing the nitrogen plasma, was performed. A corresponding experimental arrangement is presented in Fig. 1.

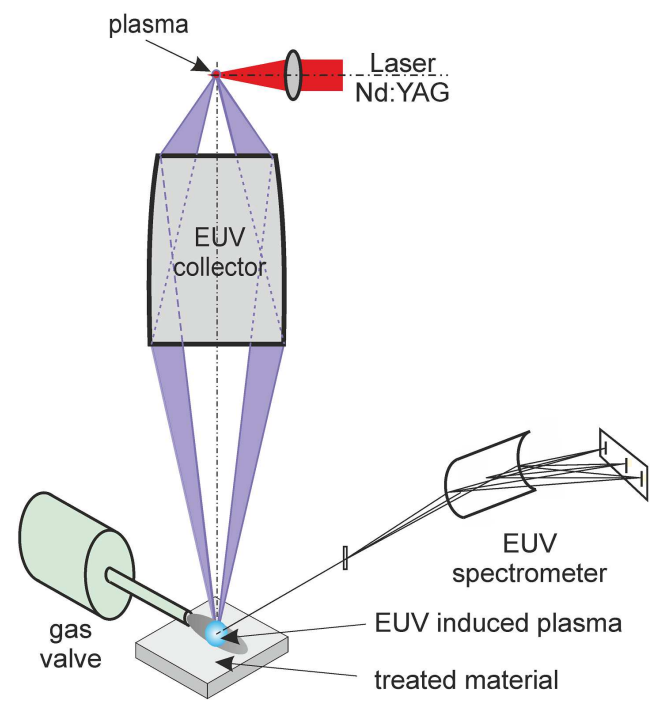

Fig. 1. Schematic view of the experimental arrangement.

In Fig. 2a an emission spectrum of N(II) ions recorded in the EUV range is presented. Measurements of the emission Vis spectra acquired for the nitrogen plasma indicate that except atomic also molecular ions are being formed. Examples of spectra obtained for $\mathrm{N}_{2}^{+}$ions are shown in Fig. 2b,c. It should be pointed out that the molecular spectra dominate within the UV-Vis range. Both single charged ions and molecular ions can react
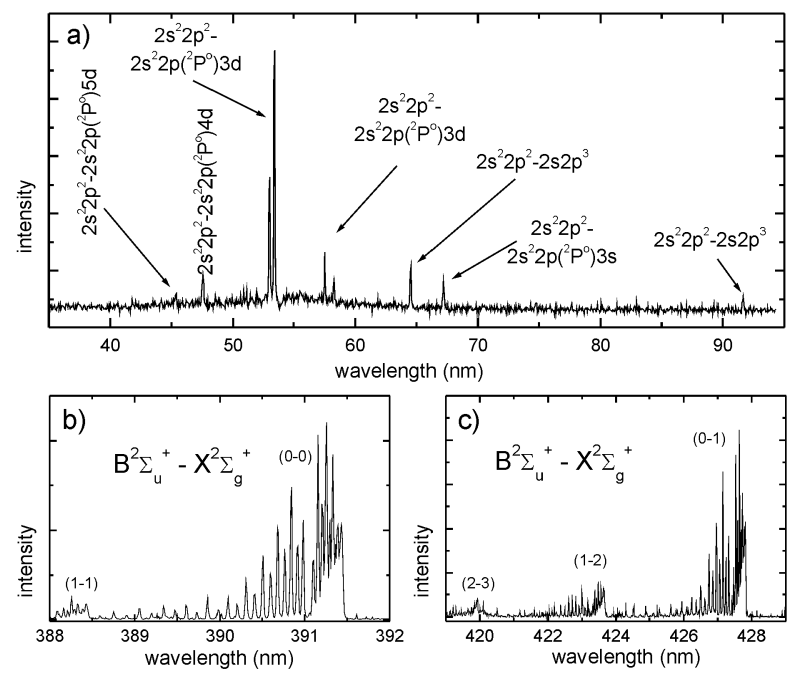

Fig. 2. Emission spectra of the low temperature photoionized nitrogen plasma: (a) N(II) ions, EUV range, $(\mathrm{b}, \mathrm{c}) \mathrm{N}_{2}^{+}$ions, Vis range.

with the irradiated material, forming new molecules or functional groups.

\section{Surface structuring}

The surface structuring concerns either creation of microstructures of defined shape (micromachining) or selforganized micro- and nanostructures formed in the nearsurface layer. The micromachining of materials, mainly polymers, can be realized by irradiation of the surface through a contact mask. Surface irradiation without any mask results in many cases in changes of surface morphology in micro- or nanoscale. In principle the EUV induced micromachining is possible for any polymer, however, irradiation of selected polymers results in smooth ablation, in other cases roughness of the surface increases during ablation. These polymers are, thus, not very suitable for micromachining. Examples of such structures created in poly(methyl methacrylate) (PMMA), poly(ethylene terephthalate) (PET) and PVDF are presented in Fig. 3. The structures were obtained by irradiation of polymer foils with multiple EUV pulses through a copper mesh. In most cases it was found that an average value of the ablation rate is approximately $100 \mathrm{~nm}$ per pulse. Micromachining of materials for which the surface after ablation remains rough is also possible. In such cases, however, RMS of the surface can be comparable with the smallest dimension of the transferred pattern. Examples of microstructures created in PET irradiated through a metallic grid with $100 \mu \mathrm{m}$ period are shown in Fig. 3c. The structures were obtained after irradiation with 300 EUV pulses.

As it was mentioned above, EUV irradiation of some materials can be associated with modification of the surface morphology. A proper adjustment of irradiation parameters can result in surface modification of any polymer. In case of some polymers, however, significant 
a)
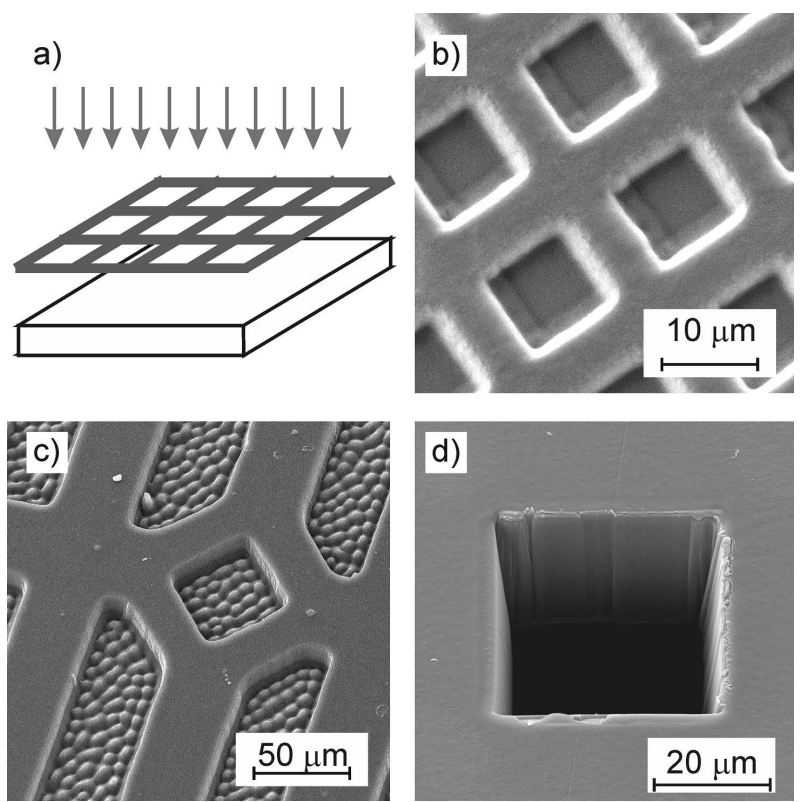

Fig. 3. Micromachining by EUV induced ablation: (a) schematic view of irradiation; surface patterns created in PMMA (b), PET (c), PVDF (d), respectively.
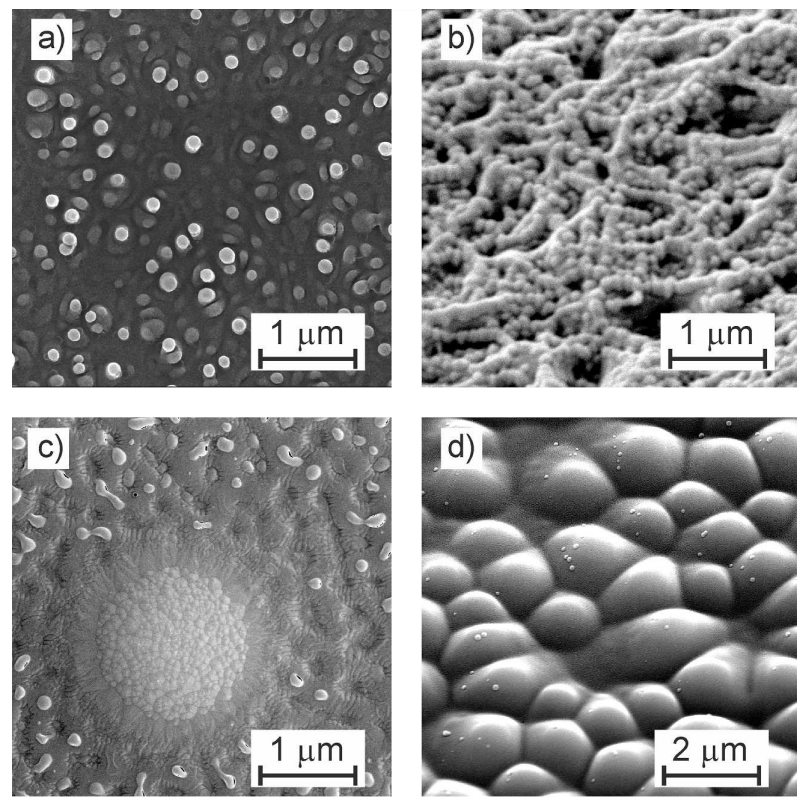

Fig. 4. SEM images of micro and nanostructures created in different materials: (a) PMMA, (b) PTFE, (c) GaAs, (d) glass.

changes in surface morphology can be obtained by irradiation with the fluence close to the ablation threshold. Apart from that usually irradiation with a large number of EUV pulses is required. In case of inorganic solids, either crystalline or amorphous, changes of the surface morphology require much higher fluence comparing to polymers. Examples of surface morphologies obtained for PMMA, polytetrafluoroethylene (PTFE), monocrystal GaAs and glass are shown in Fig. 4. In case of PMMA its surface is covered with nodules of the size 100-200 nm as shown in Fig. 4a. By removal of a several $\mathrm{nm}$ thick layer it was observed using a scanning electron microscope (SEM) that the nodules are in fact nanosized bubbles. Their origin is probably connected with a release of volatile polymer fragments trapped locally several nanometers underneath the surface. The nodules could be then created by volatilization just underneath the surface causing thereby surface swelling on the nm scale.

Quite different image was obtained for nanostructures created in the PTFE polymer. PTFE is one of the most interesting polymers used for various applications. Due to a weak absorption of the radiation from infrared (IR) to ultraviolet range, many works were devoted to micromachining of this polymer using synchrotron $\mathrm{X}$ rays $[8,9]$. On the other hand, absorption of EUV radiation in every material, including PTFE is very strong. The absorption depth is less than $100 \mathrm{~nm}$, hence, EUV induced micromachining of this material is very efficient and was demonstrated in Ref. [10]. In this work PTFE was irradiated with the fluence close to the ablation threshold. 1 min irradiation (600 EUV pulses) strongly affected the surface morphology producing cavities with the sub-microsize. 3 min irradiation results in formation of very complicated beads-on-a-string nanostructures with characteristic size of beads $50 \div 100 \mathrm{~nm}$. The structure was shown in Fig. 4b. In Fig. 4c nanostructures formed in GaAs monocrystal irradiated with 100 of EUV pulses, are presented. Their formation can be associated with melting of the near surface layer and its chemical decomposition. Except different kinds of granular structures ripples with a period of approximately $50 \mathrm{~nm}$ were formed. Their origin can be similar to laser induced periodic surface structures (LIPSS). The last image (Fig. 4d) shows the microstructure obtained for glass irradiated with relatively high fluence exceeding the value of $100 \mathrm{~mJ} / \mathrm{cm}^{2}$. In this case the material ablation was accompanied by formation of hemispherical structures with a characteristic size of approximately $1 \mu \mathrm{m}$. At the surface of such microstructures, sparsely dispersed nanoparticles are visible. Such nanoparticles were present especially in the area surrounding the interaction region. It suggests that they were ejected from the surface during the material ablation and deposited outside the irradiated region.

\section{Chemical changes}

EUV irradiation of polymers and other solids results in surface structuring. It can affect also their chemical structure. It is, however, clear that new atoms cannot be incorporated, due to irradiation in the vacuum environment. New atoms or functional groups can be incorporated in case of additional plasma treatment. In Fig. 5 four XPS spectra are presented. The first one (Fig. 5a) was obtained for a pristine PVDF sample. It contains 
two characteristic peaks corresponding to carbon (C $1 s)$ and fluorine (F 1s) atoms. Almost identical spectrum was recorded for a PVDF sample irradiated with the high fluence (Fig. 5c). In this case the efficient ablation took place and the remaining material was practically not affected. Significantly different are two other spectra. The spectrum shown in Fig. 5b was obtained for the PVDF sample irradiated with the low EUV fluence. First of all in this case the relative intensity of the F1s peak is much lower comparing to the spectra from Fig. 5a,c. It means a carbon enrichment in the near surface layer. Apart from that an additional peak - O $1 s$ is clearly visible. Oxygen atoms are not present on the pristine polymer, so they had to be incorporated during a contact of the EUV activated surface with the atmospheric $\mathrm{O}_{2}$ molecules. Similar $\mathrm{C} 1 s, \mathrm{~F} 1 s$ and $\mathrm{O} 1 s$ peaks are visible at Fig. $5 \mathrm{~d}$. In this case, however, an additional $\mathrm{N} 1 s$ peak appeared. Its presence is connected with dual action of the EUV radiation and the low temperature nitrogen plasma. The EUV irradiation breaks molecular bonds of the polymer activating the surface while the plasma delivers $\mathrm{N}^{+}$and $\mathrm{N}_{2}^{+}$ ions. It results in an effective incorporation of nitrogen atoms into the polymer molecules.
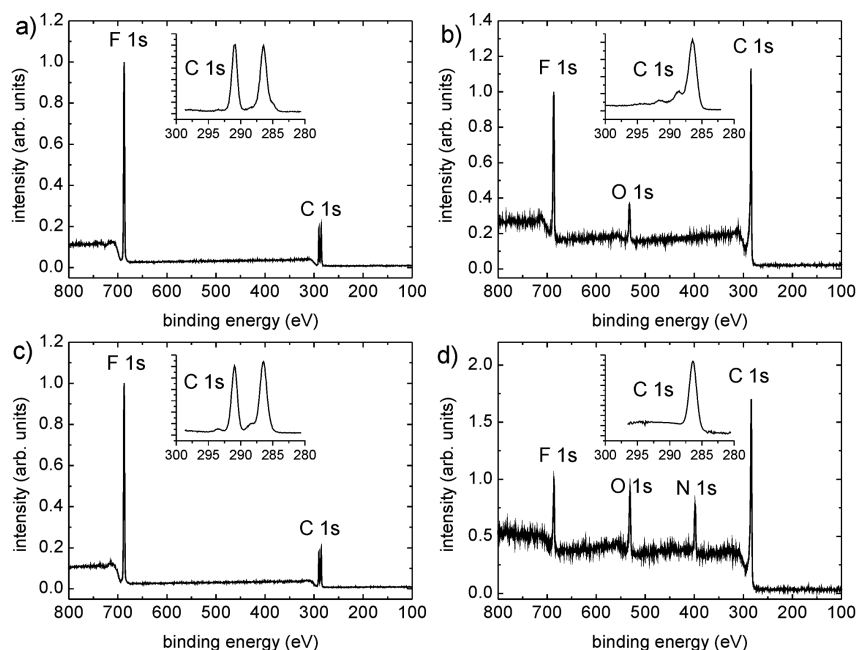

Fig. 5. XPS spectra of PVDF: (a) pristine, (b) irradiated with low EUV fluence, (c) irradiated with high EUV fluence, (d) simultaneous EUV and plasma treatment.

\section{Summary}

In this paper we presented results of experiments concerning surface structuring of solids irradiated with EUV pulses and chemical modification of polymers exposed to the EUV induced, low temperature plasmas. Results of spectral studies of the nitrogen plasma employed for the surface treatment were also presented. Different kinds of nano- and microstructures were obtained after the EUV irradiation. A possibility of efficient micromachining by the EUV induced ablation was also demonstrated. It was shown that the dual action of the EUV radiation and the nitrogen plasma allows for incorporation of $\mathrm{N}$ atoms into the polymer structure.

\section{Acknowledgments}

This work was supported by the National Science Centre, Poland, grant agreement no. UMO$2016 / 23 / \mathrm{B} / \mathrm{ST} 7 / 00949$, and partially by European Union's Horizon 2020 Programme (LASERLABEUROPE) grant agreement no. 654148.

\section{References}

[1] L. Bacakova, V. Mares, V. Lisa, V. Svorcik, Biomaterials 21, 1173 (2000).

[2] M. Collaud Coen, R. Lehmann, P. Groening, L. Schlapbach, Appl. Surf. Sci. 207, 276 (2003).

[3] T. Lippert, Adv. Polym. Sci. 168, 51 (2004).

[4] T. Gumpenberger, J. Heitz, D. Bauerle, H. Kahr, I. Graz, C. Romanin, V. Svorcik, F. Leisch, Biomaterials 24, 5139 (2003).

[5] R. Mikulikova, S. Moritz, T. Gumpenberger, M. Olbrich, C. Romanin, L. Bacakova, V. Svorcik, J. Heitz, Biomaterials 26, 5572 (2005).

[6] A. Bartnik, H. Fiedorowicz, R. Jarocki, J. Kostecki, M. Szczurek, W. Wachulak, Nucl. Instrum. Meth. Phys. Res. A 647, 125 (2011).

[7] H. Fiedorowicz, A. Bartnik, M. Szczurek, H. Daido, N. Sakaya, V. Kmetik, Y. Kato, M. Suzuki, M. Matsumura, J. Tajima, T. Nakayama, T. Wilhein, Opt. Commun. 163, 103 (1999).

[8] Y. Zhang, T. Katoh, M. Washio, H. Yamada, S. Hamada, Appl. Phys. Lett. 67, 872 (1995).

[9] T. Katoh, D. Yamaguchi, Y. Satoh, S. Ikeda, Y. Aoki, M. Washio, Y. Tabata, Appl. Surf. Sci. 186, 24 (2002).

[10] A. Bartnik, H. Fiedorowicz, R. Jarocki, J. Kostecki, M. Sawicka, M. Szczurek, Proc. SPIE 7141, 714105 (2008). 